\title{
Therapeutic Perspectives for Hepatitis C
}

\author{
OVERVIEW \\ Evaldo Stanislau Affonso de Araújo and Antonio Alci Barone \\ Laboratory of Hepatitis, LIM 47 DMIP-HC-FMUSP; São Paulo, SP, Brazil \\ NEWTHERAPIESFOR HEPATITISC \\ Jean-Michel Pawlotsky \\ French National Reference Center for Viral Hepatitis B, C and Delta; Department of Virology, \\ and INSERM U841, Hôpital Henri Mondor, Université Paris 12, Créteil, France
}

\section{Overview}

Hepatitis C virus (HCV) is represented by a single open reading frame of single-stranded RNA comprising approximately 3000 AA, positioned between 3' and 5' noncoding extremities. Transcription from the 5 ' end results in a compound polyprotein, generated by structural proteins in the amino-terminal region extremity and by nonstructural proteins in the opposing terminal region. Post-transcription cleavage by viral and host cell proteases generates 10 individual HCV proteins [1]. The HCV cDNA clone was first described in 1989. Subsequently, counter to the expectations of an enormous breakthrough, there was a hiatus in the growth of knowledge regarding the molecular virology of this virus. Only in 1997 was the first functioning and complete HCV cDNA clone described and used in animal models (chimpanzees) for preliminary analyses of the viral expression using biotechnological tools. A milestone in the study of viral molecular biology was the description, in 1999, of a viral culture model in tumor cells (Huh 7) using a HCV 1b subgenomic replicon. Based on this model, despite strict limitations, other viral fragments were used in order to evaluate the dynamics of viral replication in different settings and with exposure to antiviral agents. Nevertheless, the inability to obtain a complete HCV sequence remains an obstacle to be overcome [1].

One characteristic of HCV is that is has minimal cytopathic effects, with immunomediated hepatotropic injury. However, it is also found in extrahepatic sites, where it has a short halflife (approximately 3 hours) and high serum turnover [2]. Standard treatment involves the administration of pegylated interferon alpha together with ribavirin. This treatment regimen has had modest success, a little over 50\% [3], reaching 71\% in cases of dose optimization and full compliance [4]. However, it could be much less, if we consider real life data, as well as characteristics of the host (race, comorbidities, immune state), of the liver disease (level of fibrosis and steatosis), of the medication (dose, interferon type) and of the virus itself. In addition, it is well known that genotype 1 has a lower response rate, and certain viral proteins can subvert the stimulus induced by interferon and ribavirin [3]. Viral kinetics, the evaluation of the decay pattern of the viremia - in terms of intensity and

The Brazilian Journal of Infectious Diseases $\quad$ 2007;11 (5) Suppl. 1:73-79. (C) 2007 by The Brazilian Journal of Infectious Diseases and Contexto Publishing. All rights reserved. speed - after the initiation of the therapy, has proven to be a useful tool in the management of patients. It demonstrates the interaction among virus, drug, and host, in a relatively simple way: intense, rapid decay indicates a favorable case; insignificant, slow decay indicates cases that are unfavorable, moderate or intermediate. Such kinetic patterns allow patients to be characterized as rapid responders, slow responders, or nonresponders. This approach allows the treatment to be individualized, with ideal doses per weight and shorter, standard, or extended duration. In addition, it allows early prediction of treatment response. Therefore, individualized therapy constitutes the best therapeutic tool at the moment [5].

Of course, for the sake of effectiveness, safety, and tolerability, the current therapy is insufficient to confront the present hepatitis $\mathrm{C}$ epidemic. Therefore, from a therapy with poorly understood mechanisms of action that function through essentially indirect means [6], we have evolved to the specifically targeted antiviral therapy for hepatitis C [7], that is, the search for compounds that have a direct effect on the HCV life cycle. However, the preliminary results of current clinical studies have demonstrated worrisome aspects regarding safety, tolerability, and efficacy for some of these compounds [7], which is why we believe that treatment with interferon alpha will continue to lead the field for many years to come, and studies involving combinations of new compounds including interferon and, occasionally, ribavirin are already underway. A good example of this fact are the preliminary results of the PROVE-1 study recently presented in Barcelona [8], in which the group that used the combination of pegylated interferon alpha-2a $180 \mu \mathrm{g}+$ ribavirin 1-1.2 g + telaprevir $750 \mathrm{mg}$ every 8 hours, in an intention-to-treat analysis, showed a markedly greater response than that observed for the control group (receiving pegylated interferon + ribavirin), despite the fact that the subjects were infected with genotype 1 . At week 4, 79\% presented viremia < $10 \mathrm{IU} /$ $\mathrm{mL}$. At week 12, 70\% presented viremia $<10 \mathrm{IU} / \mathrm{mL}$, whereas $39 \%$ presented viremia $\geq 10 \mathrm{IU} / \mathrm{mL}$, emphasizing the loss of cases and the intention-to-treat analysis. Nevertheless, the incidence of rash, gastrointestinal effects, and anemia was significantly higher in the telaprevir group.

The new therapeutic options are divided into 'smallmolecule' (protease and viral polymerase inhibitors and 
protein inhibitors of the host - glucosidase) [9] - data summarized in Table 1 - and drugs whose targets are still indirect. In this second group, there are several that are currently in Phase I trials, specifically the Toll-like receptor agonists 7 and 9 (ANA 245 - Isatoribine - ANA 975 and Actilon) [10] whose mechanism of immune stimulation is broader than that induced by interferon alpha. The modulation of the inflammation and apoptosis by caspase inhibition is also under evaluation, and preliminary results demonstrate that aspartate aminotransferase normalizes in the patients who received it during trials ranging from Phase II to IDN-6556 [11]. There are other forms of interferon in phase I and II trials: albuferon (interferon alpha-2b fused to albumin, with more favorable posology regimen and similar efficacy to that of pegylated interferon); omega interferon; gamma interferon; interferon for oral use; and oral interferon inducers (resiquimod and imiquimod). In addition, there are nucleoside analogs similar to ribavirin (viramidine, now known as taribavirin) in Phase III, inosine monophosphate dehydrogenase inhibitors- VX98-497 (merimepodib) in Phase II for previous nonresponders [12], mycophenolate mofetil, also in Phase II for nonresponders, broad spectrum antiviral agents in Phase II (amantadine and rimantadine) and other immunomodulators in Phases I to III (histamine, thymosin alpha-1, IL-10 and IL-12). Finally, therapeutic vaccines are being studied in pre-clinical phases (E1/E2) I or II (E1, NS3NS4-NS5-core, IC41) [10].

After a brief review of developing drugs, we should bear in mind the problems and disappointments already encountered regarding the resistance developed in vitro or in vivo, in addition to the already mentioned weaknesses related to posology tolerance and complexity. Posology regimens, as already described for HIV, can be facilitated by the association with the protease inhibitor ritonavir, acting as a potent inhibitor of the enzyme system function of cytochrome P450, which would enable, in preliminary analyses, simplified posology regimens for some of the small molecules [13]. Regarding other problems, further review will bring up some relevant issues, in addition to the already evident necessary maintenance of interferon, and even ribavirin, in future therapeutic regimens.

\section{New Therapies for Hepatitis C}

The current standard of care for patients with chronic hepatitis $\mathrm{C}$ is the combination of pegylated interferon (IFN) alpha and ribavirin. This treatment is effective in approximately $80 \%$ of patients with hepatitis C virus (HCV) genotype 2 or 3 infection, but less than $50 \%$ of those with HCV genotype 1 [14]. Many new HCV drugs are at the preclinical developmental stage and several are in clinical development. Novel HCV therapies currently in development schematically belong to four categories: novel IFNs, alternatives to ribavirin, immune therapies, and specific and nonspecific inhibitors of the HCV lifecycle.

\section{Novel Therapies for Hepatitis C}

Novel IFNs

New IFN alpha molecules are currently being developed. They are expected to yield more potent antiviral effects, and eventually more potent immunomodulatory effects, with improved pharmacokinetic and pharmacodynamic properties and, if possible, better tolerance. The potential interest in nonalpha IFNs is also being studied.

\section{Albumin-linked IFN alpha}

Albumin-linked IFN alpha (Albuferon ${ }^{\circledR}$, Human Genome Sciences and Novartis) is an IFN alpha-2b molecule attached to a human albumin moiety which has a prolonged half-life that allows dosing at intervals of 2 to 4 weeks. Albumin-linked IFN alpha is able to induce an antiviral response in previous nonresponders to pegylated IFN and ribavirin combination. Results presented at the $42^{\text {nd }}$ Annual Meeting of the European Association for the Study of the Liver (EASL) have shown no significant difference in the rates of sustained virological response between patients receiving pegylated IFN alpha-2a and ribavirin or various doses of albumin-linked IFN alpha administered every two or four weeks with ribavirin.

\section{Consensus IFN alpha}

IFN alphacon-1 (Infergen, Amgen and InterMuneYamanouchi) is a synthetic recombinant "consensus" IFN (cIFN) created by scanning the sequences of several natural alpha IFNs and assigning the most frequently observed amino acid in each corresponding position to the recombinant molecule [15]. There is no clear evidence that cIFN is superior to other alpha IFNs when both are given in equivalent doses in terms of sustained virological response.

\section{Other alpha IFNs in development}

Multiferon (Viragen) is a highly purified, multi-subtype natural human IFN alpha derived from human leukocytes which has already been approved for use in HCV therapy in several countries. Medusa ${ }^{\circledR}$ (Flamel Technologies) is a selfassembled poly-aminoacid nanoparticles system that can be used as a protein carrier for the development of novel longacting native protein drugs. Medusa IFN alpha-2a and Medusa IFN beta are currently in early clinical development. Various types of orally administered IFNs alpha are also currently being developed. Whether similar efficacy as with subcutaneous administration can be achieved is under study.

\section{Non-type I IFNs}

IFN omega, like IFN alpha or beta, is a type 1 IFN. It shares $70 \%$ homology with IFN alpha and binds to the same receptor. A stable, glycosylated form of IFN omega has been developed (Intarcia) and has been administered to patients with chronic hepatitis $C$ in phase I and II trials. The results of a phase II trial presented at the $42^{\text {nd }}$ EASL Annual Meeting have shown sustained virological response rates (HCV RNA below 50 international units (IU)/mL at week 12 post-therapy) of 36\% in 
Table 1. Developing drugs that have a direct effect on HCV (adapted from [7,10])

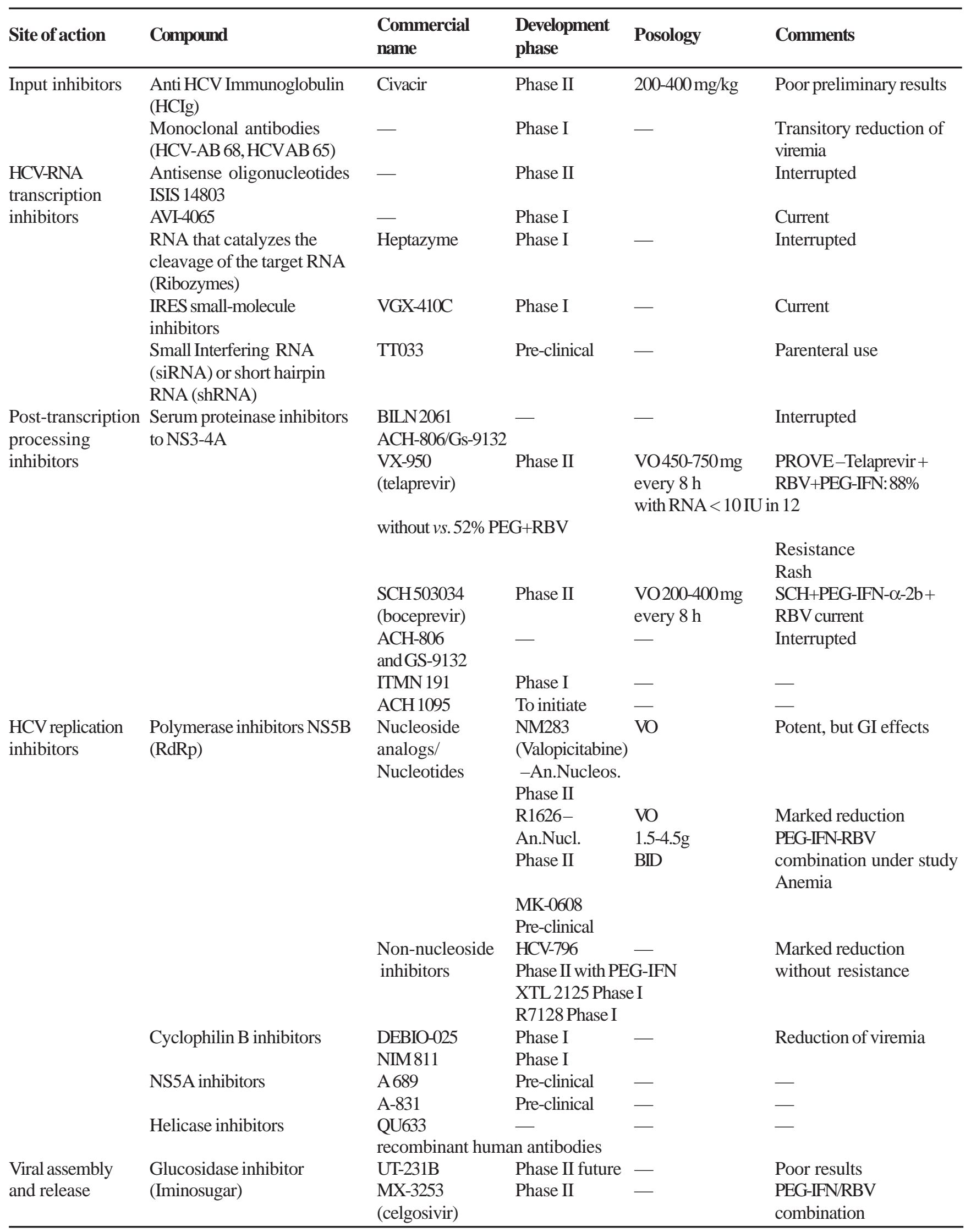


the IFN omega-ribavirin combination arm versus $6 \%$ in patients treated with IFN omega alone. The manufacturer plans to develop an implantable infusion pump that will release a steady amount of IFN omega for approximately four weeks.

In spite of in vitro results that would support a potentiating effect of IFN gamma on IFN alpha [16], the proof of concept that IFN gamma could have a utility in the treatment of chronic hepatitis $\mathrm{C}$ still remains to be made.

IFN lambda-1 (interleukin 29 or IL-29) is a member of a novel family of cytokines that are distantly related to the IL10 family and type 1 IFNs. IFN lambda- 1 exhibits dose- and time-dependent inhibition of HCV replication in various models, independent of types 1 and 2 IFN receptors and induced pathways [17]. A pegylated form of IFN lambda will soon enter clinical evaluation.

\section{Alternatives to Ribavirin}

Alternatives to ribavirin are needed that would have the same effects as ribavirin on infection in combination with pegylated IFN or other IFN molecules without its hemolytic properties. However, the challenge for discovering such molecules is hampered by the fact the antiviral mechanisms of ribavirin remain poorly understood. Taribavirin (Valeant Pharmaceuticals) is an amidine prodrug of ribavirin converted into ribavirin by adenosine deaminases, which are primarily present in hepatocytes. Taribavirin is thus preferentially taken up in the liver where it serves for ribavirin delivery to the major site of HCV replication, whereas it is not transported efficiently into red blood cells [18]. In two recent phase III trials in combination with pegylated IFN alpha 2a and 2b, respectively, taribavirin at a flat dose of $600 \mathrm{mg}$ twice a day failed to achieve similar efficacy as weight-based dosed ribavirin in patients with chronic hepatitis $C$ of various genotypes. The incidence of hemolytic anemia was however significantly lower with taribavirin. New trials with higher doses of taribavirin have been planned.

\section{Immune Therapies}

Various nonspecific immunomodulatory agents, including thymosin alpha-1 (Thymalphasin, SciClone), IL-10 or histamine (Maxym Pharmaceuticals) have been administered to patients with chronic hepatitis C, with little success.

Vaccines can induce CD4+ and CD8+ T-cell responses to $\mathrm{HCV}$. Preclinical and early human studies indicate that therapeutic vaccines using different forms of recombinant $\mathrm{HCV}$ proteins together with various adjuvants could upregulate both cellular and humoral immune responses in patients with chronic hepatitis C [19]. However, there is currently no evidence that therapeutic vaccines alone can result in changes in HCV RNA levels. It remains to be determined whether therapeutic vaccines could be useful in combination with potent antiviral molecules.

Inhibitors of the HCV Life Cycle

Every step of the HCV lifecycle constitutes a potential target for specific or nonspecific antiviral molecules. Many drugs are at the preclinical developmental stage and several are in clinical development, but initial trials using some of these inhibitors alone have raised concerns about their tolerability and the development of viral resistance. A number of specifically targeted therapies are now also being tested in combination with pegylated IFN alpha with or without ribavirin.

\section{Inhibitors of the early steps of the HCV life cycle}

Inhibition of HCV entry can be based on the use of specific antibodies that neutralize infectious particles and prevent their attachment to the receptor molecules. These include polyclonal hepatitis $\mathrm{C}$ immune globulins, that have been administered to prevent $\mathrm{HCV}$ infection in $\mathrm{HCV}$-infected liver transplant recipients with little success [20], and anti-HCV monoclonal antibodies with high-affinity HCV neutralizing properties that reduce viral replication by 0.3 to $1.0 \log [21,22]$. Small molecule entry inhibitors can theoretically belong to two groups of drugs: molecules that specifically fix onto the HCV surface structures and neutralize the virus, or molecules that compete with infectious viral particles at the receptor level. Unfortunately, our understanding of HCV entry mechanisms remains rudimentary, hampering the development of such molecules inhibitors. Fusion could also become an interesting target for novel therapies when its mechanisms are better understood.

\section{HCV RNA translation inhibitors}

Several nucleic acid-based strategies have been tested, including antisense oligodeoxynucleotides, phosphorodiamidate morpholino oligomers (PMO), or ribozymes. All of them have been shown to potently inhibit $\mathrm{HCV}$ translation in vitro, but have been disappointing in vivo [23,24]. RNA interference initiated by small interfering RNAs (siRNA) or short hairpin RNAs (shRNA) is very specific and offers a potential to be used as antiviral against HCV. However, because of their size and chemical composition, siRNAs and shRNAs currently are not orally bioavailable and require parenteral administration. Alternative strategies currently target the three-dimensional functional internal ribosome entry site (IRES) complexed with ribosomal subunits and viral and cellular proteins with small molecule inhibitors.

\section{Inhibitors of $\mathrm{HCV}$ post-translational processing}

Highly selective, potent peptidomimetic inhibitors of HCV NS3/4A proteinase have been designed. VX-950 or telaprevir (Vertex Pharmaceuticals) and SCH 503034 or boceprevir (Schering-Plough Corporation) have now advanced to phase II clinical trials. Patients who received $750 \mathrm{mg}$ of telaprevir alone every 8 hours experienced a median 4.4-log reduction in HCV RNA levels [25]. The drug is well tolerated over shortterm administration. However, viral breakthroughs occur during the second week of telaprevir administration in patients with low exposure to the drug. They are due to selection of telaprevir-resistant variants. Combination with pegylated IFN alpha with or without ribavirin could theoretically at least partly prevent telaprevir resistance. This hypothesis is currently 
under study in two phase II trials. Boceprevir appears to have less potent antiviral properties than telaprevir in vivo at the doses used to date [26]. In combination, the antiviral effect of boceprevir appears to be additive to that of pegylated IFN alpha-2b [26]. A phase II clinical trial is ongoing in combination with pegylated IFN alpha and ribavirin, where high doses of boceprevir are administered to treatment-naïve patients.

Other approaches have been developed to inhibit the NS3/ 4A serine proteinase function. ACH-806/GS-9132 (Achillion Pharmaceuticals and Gilead Sciences) inhibits binding of NS4A to the NS3 proteinase, therefore inhibiting polyprotein processing by preventing the formation of the active proteinase complex. ACH-806/GS-9132 has potent in vitro activity against HCV genotype 1 and administration of 300 mg twice daily for 5 days resulted in an average change in HCV RNA level from baseline of -0.9 log [27]. The development of this drug has been halted because of nephrotoxicity [27].

\section{Inhibitors of HCV replication}

Inhibitors of the RNA-dependent RNA polymerase (RdRp) belong to two categories: nucleoside/nucleotide inhibitors, that target the catalytic site of the enzyme, and non-nucleoside inhibitors that target allosteric sites of the RdRp. Three RdRp inhibitors have been administered to patients in clinical trials, including two nucleoside and one non-nucleoside inhibitor. Two have been withdrawn due to toxicity. R1626 (Roche Products) induces a dose-dependent HCV RNA level reduction [28]. At very high doses, HCV RNA level decrease reaches more than 3 logs, but side-effects are frequent. R1626 in combination with pegylated IFN alpha and ribavirin has recently progressed into phase II of clinical development. Other drugs have entered phase I clinical development.

The HCV RdRp has been reported to bind cyclophilin B, a cellular peptidyl-prolyl cis-trans isomerase that apparently regulates HCV replication through modulation of the RNA binding capacity of RdRp. Synthetic, non-immunosuppressive cyclophylin B inhibitors have been developed and are being tested in patients with chronic HCV infection [29]. Side-effect were however frequent and led to treatment withdrawal in several cases [16]. Another cyclophilin B inhibitor, NIM 811 (Novartis) is currently being tested in a phase I trial [30,31].

\section{Inhibitors of virus assembly and release}

Iminosugars have been suggested to be able to cross cellular membranes and concentrate in the endoplasmic reticulum where they could competitively inhibit envelope proteins glycosylation and interfere with viral assembly [32]. MX-3253 or celgosivir (Migenix) has a modest antiviral effect on HCV in monotherapy. It is currently administered in combination with pegylated IFN alpha and ribavirin in a phase II clinical trial.

\section{Questions and Issues with New Therapies}

Among the many new avenues being explored, orally administered antiviral drugs that specifically inhibit a step of the HCV lifecycle have come under the spotlight. However, although encouraging results have been published, serious issues have been raised as to the antiviral potency of these drugs, their tolerability, and the crucial problem of viral resistance.

\section{Antiviral Potency}

Antivirals usually enter development as soon as they show some degree of antiviral efficacy in one of the existing in vitro models. However, in vitro antiviral activity does not always translate into antiviral efficacy in vivo. There are several examples of drugs that were highly potent in vitro but failed when administered to patients. Unfortunately, studies with "negative" results are rarely published, even though they could teach us a lot. There are several possible reasons for these in vitro/in vivo discrepancies, including the use of poorly relevant preclinical models, misinterpretation of preclinical data, poor pharmacokinetics, poor delivery of a potentially potent drug to its target site, or the fact that the target is not physically accessible in infected cells in vivo.

\section{Tolerability}

Small-molecule viral inhibitors have been under close scrutiny for potential toxicity. The clinical development of BILN 2061 (Boehringer-Ingelheim, Ingelheim, Germany), a potent HCV NS3 serine protease inhibitor and the first drug of this type to be administered to infected patients, has been suspended because of myocardial toxicity in animals [33,34]. The development of an enormous number of potentially active HCV drug candidates was stopped before they were given to patients, owing to concerns raised by preclinical toxicity studies in vitro and in animal models. The clinical development of several drugs has been stopped because of their side-effect profile, including valopicitabine (digestive side-effects), HCV796 (ALT elevations) and ACH-806 (nephrotoxicity). Other drugs are still being developed in spite of serious side-effects. R1626 has been shown to induce a dose-dependent reduction of blood cell counts and hemoglobin levels after two weeks of administration at high doses [28], and the outcome of these effects is unknown if therapy is prolonged or if ribavirin is used in combination. Apparent safety during short-term administration does not guarantee that no serious adverse effects will occur when the drug is given for several weeks or months. Reesink et al. observed no serious adverse events during 14 days of telaprevir administration [25]. However, a recent commercial press release from Vertex Pharmaceuticals (December 13, 2006) states that, in the PROVE 1 study, a phase 2b twelve-week clinical trial of the triple combination of pegylated IFN alpha, ribavirin and telaprevir in treatment-naive patients, 3\% of the patients discontinued telaprevir because of rash (rash was the most common reason for treatment discontinuation). Drug-drug interactions may also be a problem when $\mathrm{HCV}$ inhibitors are used in combination with pegylated IFN, with or without ribavirin. In addition, although synergy or additive efficacy may be expected, antagonism 
can also occur. Here again, in vitro studies may give clues as to likely adverse effects but they cannot replace in vivo studies.

\section{Resistance}

A surprising finding has been the frequency and early timing of the emergence of resistance with all classes of antiHCV drugs. These results suggest that resistant variants are preexisting, fit, and ready to be selected by any specific HCV inhibitor. These findings disqualify HCV inhibitor monotherapy and raise major ethical issues as to whether naive or nonresponder patients should now be included in trials of these drugs in monotherapy, as there is a risk that they will be disqualified from future trials and therapies with drug combinations.

\section{Conclusion}

At this point, it is clear that specific HCV inhibitors should not be used alone. Combination therapy with oral antiviral drugs will require company portfolios to contain more than one such drug. And appropriate preclinical drug-drug interaction studies will have to be performed before clinical trials are initiated. This may take several years. In the meantime, new strategies are needed to improve the results of current HCV therapy. The "conservative“" approach aimed at optimizing pegylated interferon-ribavirin therapy should not be neglected. Preliminary results have shown that increasing the dose and/or the number of injections of pegylated IFN increases the response rate, and this is being further explored in ongoing trials. Likewise, increasing the dose of ribavirin has been shown to significantly improve the cure rate, and the adverse effects of ribavirin can now be partly controlled by using erythropoietin. Other trials are addressing the best way of tailoring the duration of treatment to the early virologic response (i.e. the HCV RNA decline at week 4 or even week 2). Another option is to enhance pegylated IFN-ribavirin efficacy by adding antiviral drugs with an additive or synergistic antiviral effect. Such combinations have the advantage of theoretically preventing the onset of resistance to the inhibitor, through the antiviral effect of IFN alpha. This may indeed happen in good IFN responders, although it is unclear whether IFN inhibition will be sufficient to avoid the emergence of resistance. In addition, patients with little or no response to IFN will in effect be receiving inhibitor monotherapy. Preliminary data are encouraging and ongoing trials will show how well double and triple combinations are tolerated, and whether the responses persist.

\section{References}

1. Tellinghusein T.L., Evans M.J., Hahn T., et al. Studying Hepatitis $C$ Virus: making the best of a bad virus. Journal of Virology 2007;81(17):8853-67.

2. Lindenbach B.D., Rice C.M. Unravelling hepatitis C virus replication from genome to function. Nature 2005;436(18):933-7.
3. Wohnsland A., Hofmann W.P., Sarrazin C. Viral determinants of resistance to treatment in patients with hepatitis C. Clinical Microbiologogy Reviews 2007;20(1):23-38.

4. Pawlotsky J.-M. Mechanisms of antiviral treatment efficacy and failure in chronic hepatitis C. Antiviral Research 2003;59:1-11.

5. Marcellin P., et al. Which patients with genotype 1 chronic hepatitis $\mathrm{C}$ can benefit from prolonged treatment with the 'accordion' regimen? J Hepatology 2007 (in press).

6. Goodbourn S., Didcock L., Randall R.E. Interferons: cell signalling, immune modulation, antiviral responses and virus countermeasures. Journal of General Virology 2000;(81):2341-64.

7. Pawlotsky J.-M., Chevaliez S., McHutchison J.G. The hepatitis C virus life cycle as a target for new antiviral therapies. Gastroenterology 2007;132:1979-98.

8. McHutchinson J.G., et al. EASL 2007 Late Breaker 786.

9. Harrison S.A. Small Molecule and novel treatments for chronic hepatitis $C$ virus infection. American Journal of Gastroenterology 2007;102:1-7.

10. McHutchinson J.G., Bartenschlager R., Patel K., Pawlotsky J.-M. The face of future hepatitis $C$ antiviral drug development: recent biological and virologic advances and their translation to drug development and clinical practice. Journal of Hepatology 2006;44:411-21.

11. Walters L., Nelson M. New therapeutic options for hepatitis C. Current Opinion on Infectious Diseases 2006;19:615-22.

12. Marcellin P., Horsmans Y., Nevens F., et al. Phase 2 study of the combination of merimepodib with peginterferon-a2b, and ribavirin in nonresponders to previous therapy for chronic hepatitis C. J Hepatology 2007 (in press).

13. Kempf D.J., Chen H.-J., Yeung J.T., et al. Pharmacokinetic boosting of VX-950, an inhibitor of HCV protease, by co-dosing with ritonavir. Journal of Hepatology 2006;44(2):S4.

14. NIH Consensus Statement on Management of Hepatitis C: 2002. NIH Consens State Sci Statements 2002;19:1-46.

15. Keeffe E.B., Hollinger F.B. Therapy of hepatitis C: consensus interferon trials. Consensus Interferon Study Group. Hepatology 1997; $26: 101 S-7 S$

16. Larkin J., Jin L., Farmen M., et al. Synergistic antiviral activity of human interferon combinations in the hepatitis $C$ virus replicon system. J Interferon Cytokine Res 2003;23:247-57.

17. Marcello T., Grakoui A., Barba-Spaeth G., et al. Interferons alpha and lambda inhibit hepatitis C virus replication with distinct signal transduction and gene regulation kinetics. Gastroenterology 2006;131:1887-98.

18. Wu J.Z., Lin C.C., Hong Z. Ribavirin, viramidine and adenosinedeaminase-catalysed drug activation: implication for nucleoside prodrug design. J Antimicrob Chemother 2003;52:543-6.

19. Leroux-Roels G., Batens A.H., Desombere I., et al. Immunogenicity and tolerability of intradermal administration of an HCV E1based vaccine candidate in healthy volunteers and patients with resolved or ongoing chronic HCV infection. Hum Vaccin 2005; $1: 61-5$.

20. Davis G.L., Nelson D.R., Terrault N., et al. A randomized, openlabel study to evaluate the safety and pharmacokinetics of human hepatitis C immune globulin (Civacir) in liver transplant recipients. Liver Transpl 2005;11:941-9.

21. Schiano T.D., Charlton M., Younossi Z., et al. Monoclonal antibody HCV-AbXTL68 in patients undergoing liver transplantation for HCV: results of a phase 2 randomized study. Liver Transpl 2006;12:1381-9.

22. Galun E., Terrault N.A., Eren R., et al. Clinical evaluation (Phase I) of a human monoclonal antibody against hepatitis $C$ virus: safety and antiviral activity. J Hepatol 2007;46:37-44.

23. McHutchison J.G., Patel K., Pockros P., et al. A phase I trial of an antisense inhibitor of hepatitis C virus (ISIS 14803), administered to chronic hepatitis C patients. J Hepatol 2006;44:88-96. 
24. Soler M., McHutchison J.G., Kwoh T.J., et al. Virological effects of ISIS 14803, an antisense oligonucleotide inhibitor of hepatitis $\mathrm{C}$ virus (HCV) internal ribosome entry site (IRES), on HCV IRES in chronic hepatitis $C$ patients and examination of the potential role of primary and secondary $\mathrm{HCV}$ resistance in the outcome of treatment. Antivir Ther 2004;9:953-68.

25. Reesink H.W., Zeuzem S., Weegink C.J., et al. Rapid decline of viral RNA in hepatitis C patients treated with VX-950: a phase Ib, placebo-controlled, randomized study. Gastroenterology 2006;131:997-1002.

26. Sarrazin C., Rouzier R., Wagner F., et al. SCH 503034, a novel hepatitis $\mathrm{C}$ virus protease inhibitor, plus pegylated interferon á-2b for genotype 1 non-responders. Gastroenterology 2007; in press.

27. Pottage J.C., Lawitz E., Mazur D., et al. Short-term antiviral activity and safety of ACH-806 (GS-9132), an NS4A antagonist, in HCV genotype 1 infected individuals. J Hepatol 2007; in press.

28. Roberts S., Cooksley G., Dore G., et al. Results of a phase 1b, multiple dose study of R1626, a novel nucleoside analogue targeting HCV polymerase in chronic HCV genotype 1 patients. Hepatology 2006;44 (suppl. 1):692A.
29. Flisiak R., Orban A., Kierkus J., et al. The cyclophilin inhibitor DEBIO-025 has a potent dual anti-HIV and anti-HCV activity in treatment-naïve $\mathrm{HIV} / \mathrm{HCV}$ co-infected subjects. Hepatology 2006;44 (suppl. 1):609A.

30. Ma S., Boerner J.E., Tiong Yip C., et al. NIM811, a cyclophilin inhibitor, exhibits potent in vitro activity against hepatitis $\mathrm{C}$ virus alone or in combination with alpha interferon. Antimicrob Agents Chemother 2006;50:2976-82.

31. Goto K., Watashi K., Murata T., et al. Evaluation of the antihepatitis $\mathrm{C}$ virus effects of cyclophilin inhibitors, cyclosporin A, and NIM811. Biochem Biophys Res Commun 2006;343:879-84.

32. Durantel D., Carrouee-Durantel S., Branza-Nichita N., et al. Effects of interferon, ribavirin, and iminosugar derivatives on cells persistently infected with noncytopathic bovine viral diarrhea virus. Antimicrob Agents Chemother 2004;48:497-504.

33. Hinrichsen H., Benhamou Y., Wedemeyer H., et al. Short-term antiviral efficacy of BILN 2061, a hepatitis C virus serine protease inhibitor, in hepatitis $C$ genotype 1 patients. Gastroenterology 2004;127:1347-55.

34. Reiser M., Hinrichsen H., Benhamou Y., et al. Antiviral efficacy of NS3-serine protease inhibitor BILN-2061 in patients with chronic genotype 2 and 3 hepatitis C. Hepatology 2005;41:832-5. 